\title{
Electron Energy Loss spectroscopy of thin slabs with supercell calculations
}

\author{
Christine Giorgetti, ${ }^{1,2, *}$ Ilia Iagupov, ${ }^{1,2}$ and Valérie Véniard ${ }^{1,2}$ \\ ${ }^{1}$ Laboratoire des Solides Irradiés, CNRS, Ecole polytechnique, \\ CEA/DRF/IRAMIS, Institut Polytechnique de Paris, F-91128 Palaiseau \\ ${ }^{2}$ European Theoretical Spectroscopy Facility (ETSF)
}

(Dated: November 24, 2020)

\begin{abstract}
Electron energy loss spectroscopy in the low loss regime is widely used to access to the screening of the Coulomb potential as a function of the momentum transfer. This screening is strongly reduced for low dimensional materials and this spectroscopy is a technique of choice to study the resulting quantum confinement. Time-dependent density functional theory within an ab initio formalism, is particularly suited to simulate angular resolved electron energy loss spectra, taking benefit from the reciprocal space description. For an isolated object, the standard procedure based on the supercell approach dramatically fails for the out-of-plane optical response of the surface and we have proposed a new scheme called Selected- $G,{ }^{1}$ leading to a slab potential. In this paper, we show that the standard procedure also affects the in-plane components of the EEL spectra. Applying the Selected- $G$ procedure, we show that the full expression of the slab potential is crucial to describe slabs of finite thickness. We compare our formalism to other cutoff procedures, and show that if they provide spectra with the correct spectral weight, allowing the good description of plasmon dispersion, the amplitude of the peaks depends on the choice of the supercell. Our results, which provide spectra independent of vacuum, will have a strong impact on the calculation of properties such as quasiparticle corrections.
\end{abstract}

\section{INTRODUCTION}

Electron energy loss (EEL) spectroscopy is a widely used technique to measure electronic properties of materials. In particular, in the low loss regime, it gives access to plasmons leading to the screening of the Coulomb interaction. $^{2}$ Therefore, it has been applied to low dimensional objects, like nanosphere, ${ }^{3}$, nanotubes,${ }^{3-6}$ or thin-layer systems ${ }^{7-13}$, where the screening is strongly modified as compared to the bulk counterpart.

A dedicated framework for the theoretical description of spectroscopy in bulk materials is based on reciprocal space, as it is intimately associated to the crystalline nature of materials described historically by solid state physics. Indeed, the 3D-periodicity of the crystal and the Bloch theorem naturally suggest the use of a plane wave basis set. Therefore, time-dependent density functional theory (TDDFT) in reciprocal space is a tool of choice to calculate angular-resolved electron energy loss spectra, within an ab initio formalism ${ }^{14,15}$. It allows the transformation of the equations describing the response of the matter to a perturbation, which are originally integrals over space variables, into matrix equations, which can easily been solved numerically. This is the reason why this formalism is so powerful.

When dealing with $1 \mathrm{D}$ or $2 \mathrm{D}$ isolated objects, one loses the periodicity, in at least one direction. A way to treat this situation is to use a mixed-space formalism: for example in the $2 \mathrm{D}$ case, the reciprocal space in the plane and the real space out-of-plane $(z)^{11,13,16,17}$. It is nevertheless computationally very demanding, since it requires small integration steps along the $z$ axis $^{1,18}$.

The remaining periodicity justifies the use of 3Dreciprocal space codes. The standard procedure is to build a supercell, which plays the role of the unit cell, repeated in the three real space directions. The super- cell is composed of matter and vacuum to isolate the artificial replicas. ${ }^{19-22}$ Ground state properties are well described within this framework. ${ }^{20,23}$ For spectroscopic properties like plasmons for in-plane momentum transfers, such a formalism has been applied and can reproduce experimental results, under the condition that the supercell is large enough. ${ }^{24} \mathrm{~A}$ work dealing with excitons has recently been published. ${ }^{25}$ In this framework, cutoff procedures have been develop to isolate repeated slabs at much shorter distance, ${ }^{26,27}$ and the definition of the $2 \mathrm{D}$ macroscopic constant has also been reconsidered. ${ }^{28-30}$

Nevertheless, we have shown recently that such a formalism fails for the absorption spectrum of surfaces, in particular for the out-of-plane component when one accounts for the local fields. ${ }^{1}$ We have demonstrated that the result given by the standard supercell approach is equivalent to an effective medium theory with vacuum, and leads non physical results. To cure this vacuum problem, we have proposed, within TDDFT, a formalism called Selected- $G$, where (i) the response functions are expanded on a new set of $3 \mathrm{D}$ reciprocal lattice vectors: the new set is defined according to the thickness of the matter rather than the thickness of the supercell, (ii) due to the finite thickness of the slabs, the Coulomb interaction in the Dyson equation has to be modified (slab potential). This formalism was applied, in the limiting case of surfaces, where due to the infinite thickness of the slab, the slab potential tends towards the standard $3 \mathrm{D}$ potential, and successfully simulates the absorption spectra as well as second harmonic generation spectra for different silicon surfaces. ${ }^{1,31}$

In this paper, we apply the Selected- $G$ formalism, using the slab potential, to simulate EEL spectra of thin slabs of stacked graphene. In the first part, we summarize the procedure used to calculate the EEL spectra, within TDDFT. In the second part, we show that the in- 
plane components of the EEL spectra are also affected by the vacuum introduced in the supercell, when calculated with the standard supercell approach. The Selected- $G$ method is used to solve this spurious dependence on vacuum, and we emphasize the importance of the slab potential to account for the finite thickness of the slabs. In the third part, we compare our method with other formalisms using cutoff procedures, ${ }^{26,27}$, or slab potentials .${ }^{17,32}$ In the last part, we compare our results to the pioneering EEL measurements of few layers graphene slabs of T. Eberlein et $a l^{7}$, and show that within this framework, our numerical results reproduce the measurements, and do not depend on the amount vacuum introduced in the supercell, in contrast to previous calculations.

\section{TDDFT FORMALISM: FROM 3D TO 2D SYSTEM.}

\section{A. Dielectric function evaluated in TDDFT}

In the case of bulk (3D) materials, the reciprocal space basis vectors are built using the volume of the unit cell, while the volume of the supercell is used for lowdimensional materials.

The Dyson equation ${ }^{15}$, in reciprocal space, is a matrix equation, and reads in the random phase approximation (RPA):

$$
\begin{aligned}
& \chi_{\mathbf{G G}^{\prime}}(\mathbf{q} ; \omega)=\chi_{\mathbf{G G}^{\prime}}^{0}(\mathbf{q} ; \omega)+ \\
& \sum_{\mathbf{G}_{1}} \chi_{\mathbf{G G}_{1}}^{0}(\mathbf{q} ; \omega) v_{\mathbf{G}_{1}}(\mathbf{q}) \chi_{\mathbf{G}_{1} \mathbf{G}^{\prime}}(\mathbf{q} ; \omega) .
\end{aligned}
$$

Atomic units are used throughout unless otherwise stated. $\mathbf{q}$ is a reciprocal lattice vector which spans the first Brillouin zone, and $\omega$ the frequency. $\chi$ is the full susceptibility, corresponding to the response to the external potential and $\chi^{0}$ is the so-called independent-particle (Kohn-Sham) response function, describing the response to the total (external + induced) potential. The differ- ence between these two quantities arises from the microscopic structure of the material, as the macroscopic external perturbation leads to a microscopic electronic induced density. This phenomenon describes the so-called local field effects linked to the microscopic components $(\mathbf{G} \neq \mathbf{0})$ of the the Coulomb potential: ${ }^{33-35}$

$$
v_{\mathbf{G}}(\mathbf{q})=\frac{4 \pi}{|\mathbf{G}+\mathbf{q}|^{2}} .
$$

Once $\chi_{\mathbf{G G}^{\prime}}(\mathbf{q} ; \omega)$ is calculated, the microscopic inverse dielectric function is evaluated, according to:

$$
\varepsilon_{\mathbf{G G}^{\prime}}^{-1}(\mathbf{q} ; \omega)=\delta_{\mathbf{G G}^{\prime}}+v_{\mathbf{G}}(\mathbf{q}) \chi_{\mathbf{G G}^{\prime}}(\mathbf{q} ; \omega) .
$$

and the loss function is proportional to the imaginary part of the inverse dielectric function $-\Im\left(\varepsilon^{-1}\right)$.

We have shown that, for 2D materials described within a supercell approach, this formalism behaves as an effective medium theory, and provides unphysical absorption spectrum for the out-of-plane component of the surface. ${ }^{1}$

\section{B. Selected- $G$ approach}

To avoid this problem, we can solve the matrix Dyson equation in the 3D-reciprocal space, but using a reduced set of reciprocal lattice vectors $\tilde{\mathbf{G}}=\left(\mathbf{G}_{\|}, \tilde{G}_{z}\right)$, where $\tilde{G}_{z}$ is defined in terms of $2 \pi / L_{z}^{\text {mat }}, L_{z}^{\text {mat }}$ being the height of the matter and not the supercell. This formalism has been developed in Ref. [1] and is summarized in appendix A. Equation (1) becomes:

$$
\begin{aligned}
& \tilde{\chi}_{\tilde{\mathbf{G}}^{\prime} \tilde{\mathbf{G}}^{\prime}}(\mathbf{q} ; \omega)=\chi_{\tilde{\mathbf{G}} \tilde{\mathbf{G}}^{\prime}}^{0}(\mathbf{q} ; \omega) \\
& +\sum_{\tilde{\mathbf{G}}_{1} \tilde{\mathbf{G}}_{2}} \chi_{\tilde{\mathbf{G}}^{\prime} \tilde{\mathbf{G}}_{1}}(\mathbf{q} ; \omega) \tilde{V}_{\tilde{\mathbf{G}}_{1} \tilde{\mathbf{G}}_{2}}(\mathbf{q}) \tilde{\chi}_{\tilde{\mathbf{G}}_{2} \tilde{\mathbf{G}}^{\prime}}(\mathbf{q} ; \omega) .
\end{aligned}
$$

Due to the finite extension of the matter, the Fourier transform of the Coulomb potential depends on two variables $\tilde{\mathbf{G}}_{\mathbf{1}}, \tilde{\mathbf{G}}_{\mathbf{2}}$, which have the same in-plane projection:

$$
\begin{aligned}
& \tilde{V}_{\tilde{\mathbf{G}}_{\mathbf{1}} \tilde{\mathbf{G}}_{\mathbf{2}}}(\mathbf{q})=\frac{4 \pi}{\left|\mathbf{G}_{\mathbf{1}}+\mathbf{q}\right|^{2}} \delta_{\tilde{\mathbf{G}}_{1} \tilde{\mathbf{G}}_{\mathbf{2}}}+\frac{\xi 4 \pi \delta_{\mathbf{G}_{\mathbf{1}}, \mathbf{G}_{\mathbf{2}} \|}}{\left|\mathbf{q}+\tilde{\mathbf{G}}_{\mathbf{1}}\right|^{2}\left|\mathbf{q}+\tilde{\mathbf{G}}_{\mathbf{2}}\right|^{2}} \times \\
& \left\{-\left(2 q_{z}+\tilde{G}_{z 1}+\tilde{G}_{z 2}\right) \frac{e^{-\left|\mathbf{G}_{\mathbf{1}}\right|+\mathbf{q}_{\|} \mid L_{z}^{\text {mat }}} \sin \left(q_{z} L_{z}^{m a t}\right)}{L_{z}^{m a t}}+\frac{\left|\mathbf{G}_{\mathbf{1} \|}+\mathbf{q}_{\|}\right|^{2}-\left(q_{z}+\tilde{G}_{z 1}\right)\left(q_{z}+\tilde{G}_{z 2}\right)}{\left|\mathbf{G}_{\mathbf{1} \|}+\mathbf{q}_{\|}\right|} \frac{e^{-\left|\mathbf{G}_{\mathbf{1} \|}+\mathbf{q}_{\|}\right| L_{z}^{\text {mat }}} \cos \left(q_{z} L_{z}^{\text {mat }}\right)-1}{L_{z}^{\text {mat }}}\right\}
\end{aligned}
$$

$\mathbf{q}_{\|}$and $q_{z}$ are respectively the in-plane and out-of-plane projection of $\mathbf{q}$, in the first Brillouin zone. $\xi$ is a phase factor, defined as $\xi=1$ if the matter is located in $\left[-L_{z}^{\text {mat }}, 0\right]$ or $\left[0, L_{z}^{\text {mat }}\right]$ inside the supercell and $\xi=(-1)^{n_{1}+n_{2}}$ if it is located in $\left[-L_{z}^{\text {mat }} / 2, L_{z}^{\text {mat }} / 2\right], n_{i}$ being related to $\tilde{G}_{z i}$ by $\tilde{G}_{z i}=n_{i} 2 \pi / L_{z}^{\text {mat }}$, (see App. A 1 ).

The first term of the Slab potential (Eq. 5) is the usual 3D Fourier transform of the Coulomb potential.
The second term, non-diagonal for $\tilde{G}_{z i}$, is a correction accounting for the finite thickness of the matter, as it can be seen from the $1 / L_{z}^{m a t}$ factor. When one deals with surfaces, one considers the limit $L_{z}^{\text {mat }} \rightarrow \infty$, and the potential is reduced to the first (3D) diagonal term.

For the case of finite thickness slabs, the expression of the microscopic inverse dielectric matrix must be modi- 
fied with respect to Eq. (3) and reads now:

$$
\varepsilon_{\tilde{\mathbf{G}} \tilde{\mathbf{G}}^{\prime}}^{-1}(\mathbf{q} ; \omega)=\delta_{\tilde{\mathbf{G}} \tilde{\mathbf{G}}^{\prime}}+\sum_{\tilde{G}_{z 1}} \tilde{V}_{\tilde{\mathbf{G}}^{\mathbf{G}} \tilde{\mathbf{G}}_{1}}(\mathbf{q}) \tilde{\chi}_{\tilde{\mathbf{G}}_{1} \tilde{\mathbf{G}}^{\prime}}(\mathbf{q} ; \omega)
$$

Similar expressions have been obtained both for the slab potential (Eq. 5) and for the microscopic inverse dielectric matrix (Eq. 3) in Refs. [17, 32].

A potential based on the same idea was proposed in Ref. [30]. The off-diagonal correcting term is similar to the one proposed in Refs. [17,32], but the head of the potential $\tilde{V}_{\mathbf{0 0}}(\mathbf{q})$ is set to 0 .

The fundamental difference between these frameworks and ours is the choice for the reciprocal space basis vectors. They keep the basis built on the full supercell, when we use the Selected- $G$ one. This point will be discussed later in Part III D.

\section{RESULTS AND DISCUSSION}

The ground state properties are calculated within density functional theory (DFT) in the local density approximation (LDA), using the Abinit code ${ }^{36}$. We used a normconserving Troullier-Martins pseudopotential, with a cutoff energy of $31 \mathrm{Ha}$. The independent-particle response function $\chi^{0}$ is calculated as the sum over Kohn-Sham states, using the DP code $^{37}$. This code also solves the Dyson equations (Eq. 1) or (Eq. 4) and evaluates the microscopic inverse dielectric matrix $\varepsilon^{-1}$ using (Eq. 3) or (Eq. 6) accordingly. For small momentum transfer q, the EEL spectrum is given by $-\Im\left[\varepsilon_{\mathbf{0 0}}^{-1}(\mathbf{q} ; \omega)\right]$.

The system chosen is a AB stacking of 4 graphene layers. The thickness of the stacked graphene layers is defined as $N \times L_{z}^{\text {graphene, }}$, where $\mathrm{N}$ is the number of layers and $L_{z}^{\text {graphene }}$ the thickness of the graphene sheet. ${ }^{38}$ $L_{z}^{\text {graphene }}$ usually corresponds to half of the graphite unit cell along the c axis, which gives $3.354 \AA$. In this work, we consider the height of the supercell after atomic relaxation, leading to $L_{z}^{\text {graphene }}=3.331 \AA=6.294 \mathrm{Bohr}$. Note that this value is close to the van der Waals atomic radius. ${ }^{39}$ However, since the numerical value of this important quantity cannot be assigned unambiguously wihin a range smaller than a few percent, we have checked that it has a negligible influence on our results within a variation of $L_{z}^{\text {graphene }}$ up to $17 \%$ (see appendix B).

The thickness of the 4 layer-graphene is then $L_{z}^{m a t}=25.18$ Bohr. This slab is introduced in supercells of different heights $L_{z}^{\text {supercell }}$, defined as multiple of the height of the matter $L_{z}^{\text {mat }}$. $R$ refers to the ratio between the height of the supercell and the height of the matter: $R=L_{z}^{\text {supercell }} / L_{z}^{\text {mat }}$. The $k$-points grid is $40 \times 40 \times 1$, with parameters given in table (I). EEL spectra are calculated for an in-plane vanishing momentum transfer.

\begin{tabular}{lcccc}
\hline \hline Parameters & 4L (R2) & 4L (R3) & 4L (R4) & 4L (R5) \\
\hline npwwfn & 3451 & 5191 & 6931 & 8659 \\
nbands & 192 & 192 & 192 & 192 \\
npwmat $_{x y}$ & 19 & 19 & 19 & 19 \\
npwmat $_{z}$ & 49 & 73 & 97 & 121 \\
npwmat $_{z}$ (SG) & 25 & 25 & 25 & 25
\end{tabular}

TABLE I. Parameters used to calculate EEL spectra of the 4-layers graphene slab as a function of the supercell.

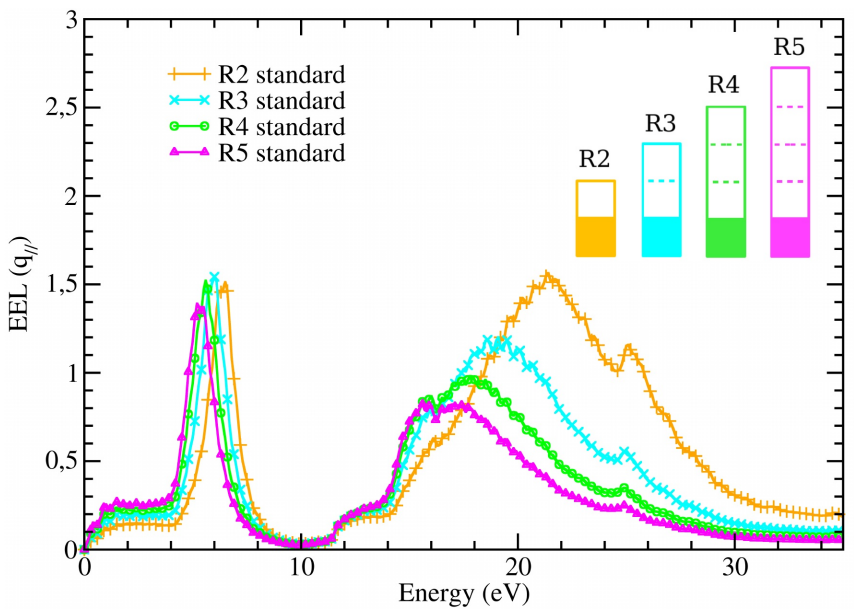

FIG. 1. (color online) Comparison of EELS spectra, including LF, for a slab of 4 graphene layers introduced in different supercells (see inset), using the standard supercell approach (Equations 1 and 3 ). $R$ refers to the ratio between the height of the supercell and the height of the matter: $R=L_{z}^{\text {supercell }} / L_{z}^{\text {mat }}$. (Orange): $\mathrm{R} 2$ - solid line with + , (Cyan): R3 - solid line with $\times$, (Green): R4 - solid line with open circle, (Magenta): R5 - solid line with up triangle. The spectra are calculated within TDDFT, for an in-plane $q_{\|}=10^{-2}$ a.u..

\section{A. Standard supercell calculations}

The results calculated using the standard supercell approach, including LF (Eq. 1 and 3), are presented in Figure (1): it is clear that the spectra, energy position and amplitude of the $\pi$ and $\pi+\sigma$ plasmons depend on the amount of vacuum introduced in the supercell.

This is a noticeable difference with the results obtained for the absorption spectra of silicon surfaces ${ }^{1}$. In that case, the energy position of the absorption peaks were unchanged for the in-plane components and the amplitude was scaled by the factor $1 / R$. For the out-of-plane component, a shift in energy and a reduction of the amplitude were present, leading to a nonphysical absorption spectrum. These results are explained by the fact that absorption can be also evaluated by a slightly different Dyson equation, ${ }^{35}$ where the long-range component of the Coulomb potential in Eq.(1) is suppressed $\left(v_{\mathbf{0}} \equiv 0\right)$. Therefore, in-plane absorption, with small LF effets, is given by $\chi^{0}$, which is just proportional to $1 / L_{z}^{\text {supercell }}$.

The situation is different for the EEL spectra, since 
they are governed by the susceptibility given in Eq. (1). In that case, the long-range component of the Coulomb potential is always present and dominates for small momentum transfer. The difference between $\chi$ and $\chi^{0}$, resulting from the presence of $v_{0},{ }^{35}$ explains why the shift in energy occurs also for the in-plane components, and is almost independent of the inclusion of LF for the considered momentum transfer $q_{\|}=10^{-2}$ a.u.. Indeed, the EEL spectra without LF (not shown) are very similar to the ones of Fig. (1).

The EEL spectrum for the largest supercell (R5) in Fig.(1) is quite close to the experimental results presented in Ref. [7]. Such a behavior was also observed when simulating the EEL spectra of graphene in the supercell formalism. Once the supercell is large enough, all the spectra are similar, apart from a normalisation factor $1 / L^{\text {supercell }} .{ }^{24}$ In that case, the good agreement of the plasmon energy compared to experimental results explains the success of this framework to study $2 \mathrm{D}$ materials, even if the large size of the supercells required to isolate the replicas has motivated the further developments.

Nevertheless, even if the energy position of the peaks can be considered as correct, the amplitude of the calculated spectra depends strongly on the size of the supercell. For this reason, it is interesting to apply the Selected- $G$ formalism for the case of EELS.

\section{B. Selected- $G$ scheme in the limit $L_{z}^{\text {mat }} \rightarrow \infty$.}

Before testing the influence of the off-diagonal corrections of the slab potential, we will first analyze the EEL spectra calculated using the Selected- $G$ formalism, with only the first term of the slab Coulomb potential (Eq. 5 ), as it is the case for surface or thick slab calculations. Since it is equal to the usual expression of the 3D Fourier transform of the Coulomb potential, it will be denoted $V_{3 D}$. The results are shown in Fig. (2). In that case, the double summation appearing in the Dyson equation (Eq. 4) is reduced to a single sum like in (Eq. 1), and the expression of the microscopic inverse dielectric matrix (Eq. 6 ) recovers the expression of (Eq. 3). The only difference with the standard supercell formalism lies in the fact that all the quantities are defined on the reduced set of $\{\tilde{\mathbf{G}}\}$ vectors.

The results are presented in Fig. (2) using the same supercells as in Fig. (1) with the same color code. Note that when using the 3D expression for the Fourier transform of the Coulomb potential, the number of bands has to be increased up to 240 bands for convergence above $35 \mathrm{eV}$. As already shown for the optical response of surfaces, all the spectra are superimposed, confirming that the Selected- $G$ procedure leads to EEL spectra independent of the amount of vacuum. Nevertheless, the resulting spectrum does not correspond to the EELS of the 4 graphene layers' slab. Indeed, the spectra are very different from the ones measured by Eberlein $e a^{7}$, where the $\pi+\sigma$ plasmon is strongly depressed above $20 \mathrm{eV}$. In the present case, the resulting spectrum corresponds actually to the bulk graphite, which is plotted in black circles in Fig. (2) for comparison.

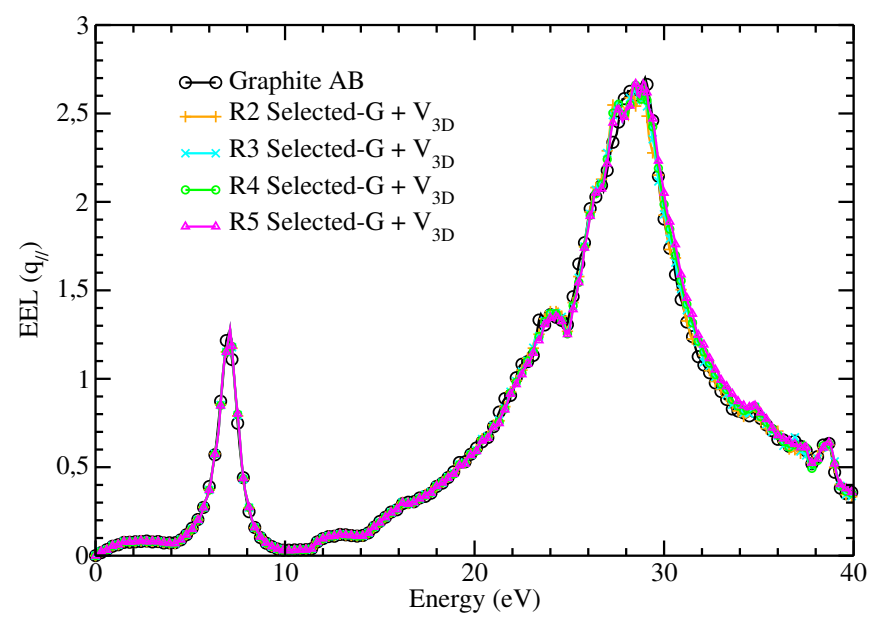

FIG. 2. (color online) Comparison of EELS spectra, including LF, for a slab of 4 graphene layers introduced in the same supercells as in Fig. (1), using the Selected- $G$ method with only the first term $\left(V_{3 D}\right)$ of the slab potential. (Orange): R2 solid line with,$+($ Cyan): R3 - solid line with $\times$, (Green): R4 - solid line with open circle, (Magenta): R5 - solid line with up triangle. The spectra are calculated within TDDFT, for an in-plane $q_{\|}=10^{-2}$ a.u. (Black) bulk graphite for reference.

\section{Selected- $G$ scheme with the slab potential.}

We will now show the EELS for these four supercells, using the Selected- $G$ procedure with the slab potential (Eqs. 4, 5, 6). Results are presented in Fig. (3), using the same color code.

As expected from the previous section, the spectra in Fig. (3) are all superimposed, leading to a result independent of the vacuum introduced in the supercell, but the resulting spectrum does not correspond anymore to the EELS of bulk graphite. The $\pi$ plasmon located above $5 \mathrm{eV}$ in the bulk case, is slightly shifted below $5 \mathrm{eV}$ for the 4 layers' slab, but has a similar amplitude. The major difference comes from the $\pi+\sigma$ plasmon. It is strongly depressed for the energy ranging between 20 and $40 \mathrm{eV}$, leading to a maximum around $15 \mathrm{eV}$, when it is located around $27 \mathrm{eV}$ for the bulk case (See Fig. 2). The spectrum calculated for the slab, using the Selected- $G$ procedure with the slab potential, exhibits the strong reduction of the screening expected for a thin slab of matter, as compared to the bulk. This spectrum compares also very well with the EELS spectra of Eberlein $\mathrm{et} \mathrm{al}^{7}$, as it will be studied in detail in Part IIIE.

\section{Comparison with cutoff procedures}

The slab potential used in the present work has been obtained by Fourier transform of the Coulomb poten- 


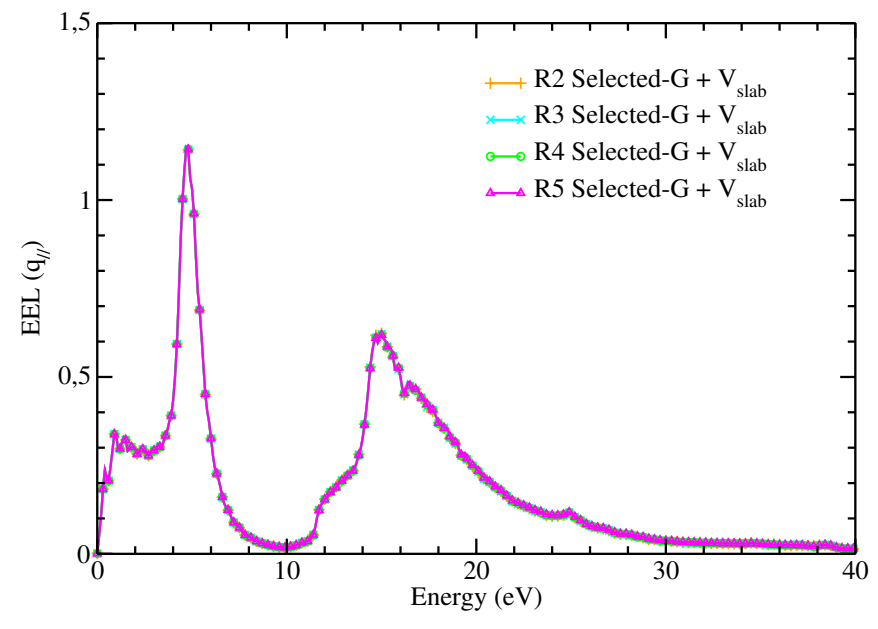

FIG. 3. (color online) Comparison of EELS spectra, including $\mathrm{LF}$, for a slab of 4 graphene layers introduced in the same supercells as in Fig. (1), using the Selected- $G$ method with the slab potential (Eq. 5). (Orange): R2 - solid line with +, (Cyan): R3 - solid line with $\times$, (Green): R4 - solid line with open circle, (Magenta): R5 - solid line with up triangle. The spectra are calculated within TDDFT, for an in-plane $q_{\|}=10^{-2}$ a.u..

tial along a finite distance, restricted to the size of the material slab and can be seen, in a way as a cutoff potential. It is thus interesting to compare our results with other standard cutoff procedures, built to tackle the problem of isolating objects in a $3 \mathrm{D}$ periodic formalism. In 2006, two papers simultaneously proposed to cutoff the Coulomb interaction at some distance between the repeated objects. ${ }^{26,27}$ In these two papers, the authors considered a diagonal expression of the Fourier transform of the 3D Coulomb potential. Note that it cannot be used for $\mathbf{q}_{\|}+\mathbf{G}_{\|}=0$, which is also the case for the slab potential we have developed.

The important point we want to focus on is the choice of the cutoff distance. In Ref. [27], the cutoff distance $z_{c}$ has to be larger than the extension of the electronic density. The size of the supercell is then deduced from this cutoff distance $z_{c}$, to avoid interaction between replicas. The minimum value corresponds to $L_{z}^{\text {Supercell }}=2 z_{c}$ for the 2D geometry. In Ref. [26], the same value was proposed, as this choice allows to cancel a divergence in the expression of the cutoff potential.

In 2013, simultaneously with our development of the Selected- $G$ procedure, leading to the slab potential (Eq. $5)^{1}$, a similar expression has been proposed ${ }^{17,32}$. It results also from the finite integration in the $z$ direction when calculating the Fourier transform of the Coulomb potential considered as a 2 -variable quantity $\left(v\left(\mathbf{r}, \mathbf{r}^{\prime}\right)\right.$ instead of $\left.v\left(\mathbf{r}-\mathbf{r}^{\prime}\right)\right)$.

However, in these approaches, the reciprocal space vectors are built considering the supercell volume. For this reason, the results still depend on the amount of vacuum introduced in the calculation, as it can be seen from Fig. 4. The parameters used for the calculations are the ones reported in table (I). In this figure, we plot the results of the cutoff procedure proposed in Refs. [26, 27] (top panel), the cutoff procedure proposed in Refs. [32, 17] (center panel) and the results of the Selected-G with the slab potential formalism (bottom panel). The left column corresponds to spectra calculated for an in-plane momentum transfer of $q=1.10^{-2}$ a.u. and the right column for $q=0.157$ a.u.. The black continuous curves corresponds to the 4 layer graphene slab in the supercell R2, and the red crosses to the spectra for the 4 layer graphene slab in the supercell R4.

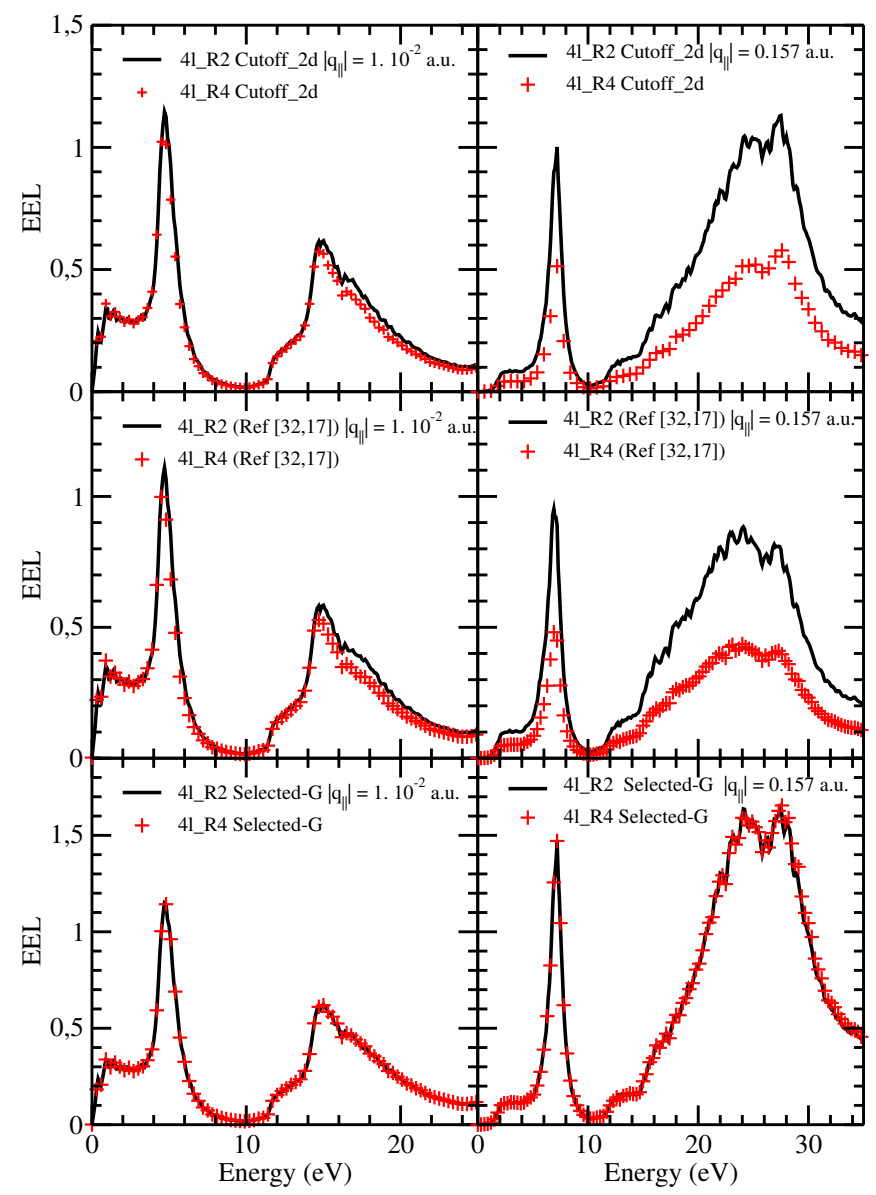

FIG. 4. (color online) Illustration of the vacuum effect for the different cutoff procedures on a 4 layer-graphene slab introduced into two supercells: ratio 2 (black continuous line) and ratio 4 (red crosses). (Top panel) cutoff proposed in Ref. [26] or [27]; (center panel) cutoff procedure proposed in Ref. [32] or [17]; (Bottom panel) Selected-G + slab potential formalism. In-plane momentum transfer: (Left column) $q_{\|}=1.10^{-2}$ a.u. and (right column) $q_{\|}=0.157$ a.u..

In the top and center panels, the calculations using either the R2- or R4-supercells lead to the same peak positions, but the amplitude of the peaks can be strongly affected. See for instance the case $q=0.157$ a.u., where the ratio between the two curves is almost equal to 2 , which corresponds to the ratio of the supercell's heights.

For small $q$, the local field effects are vanishingly small and the spectra can be evaluated from $\varepsilon_{\mathbf{0 0}}^{-1, N L F}=$ $1+V_{\mathbf{0 0}} \chi_{\mathbf{0 0}}^{0} /\left[1-V_{\mathbf{0 0}} \chi_{\mathbf{0 0}}^{0}\right]$, where $V_{\mathbf{0 0}}$ is replaced by the long 
range term $\hat{v}_{0}$ for the diagonal cutoff case. ${ }^{26,27}$. For vanishing in-plane $q_{\|}, V_{\mathbf{0 0}}\left(\right.$ or $\left.\hat{v}_{\mathbf{0}}\right) \rightarrow 2 \pi L_{z}^{S C} / q_{\|}$. Since $\chi_{\mathbf{0} 0}^{0}$ is proportional to $1 / L_{z}^{S C}$, one has an exact compensation of the dependence in $L_{z}^{S C}$.

The influence of the microscopic terms will play a role for larger momentum transfer. When $q_{\|}$increases, the compensation does not occur anymore, and the amplitude of the spectra evolves gradually towards a scaling factor corresponding to the ratio of the supercell's height. Finally, the bottom panel of Fig. (4) shows the results of the Selected- $G$ procedure with the use of the slab potential: the results are independent of vacuum, not only for vanishing $q$, as it was already evidenced in Fig. (3), but also for non-vanishing $q$ (Fig. 4 - bottom-right). Interestingly, the shape of the spectra obtained with the three different methods is quite similar. Only the amplitude is affected.

The different cutoffs proposed previously ${ }^{17,26,27,32}$ allow a correct description of the spectral weight of the EELS, which explains their success when following the dispersion of the $\pi$ and $\pi+\sigma$ plasmons, in particular in Refs. [32, 40], or 17). The common feature of all these methods is the strong reduction of the Coulomb potential, in particular of the long-range term, due the cutoff procedures. It has for consequence to reduce the amplitude of the dielectric function through Eq. (6). This effect is also obtained by increasing the size of the supercell $L_{z}^{S C}$ which normalizes $\chi^{0}$, when the calculation is done without the truncation of the Coulomb interaction.

Nevertheless, the question of the absolute amplitude of the spectrum is still open and cannot be clarified by comparing with the experimental results, since, to our knowledge, they are shown in arbitrary units. This point was already raised for the case of the diagonal cutoff, where a different definition of $\varepsilon^{-1}$ for the EELS was proposed, ${ }^{24}$, as it is discussed in the appendix $\mathrm{C} 1$.

However, the accurate determination of the amplitude is an important issue for the calculation of the screened Coulomb potential, used for example in the self-energy, at the basis of quasiparticle corrections, as well as for the absorption spectra with the Bethe-Salpeter equation formalism. Our formalism is the only one to provide quantities independent of the choice of the supercell.

\section{E. Slabs of different thicknesses}

After having discussed and solved the dependence of the spectra on the size of the vacuum in the supercell and taken into account the finite thickness of the matter, we turn now to a detailed comparison of our results with the experimental spectra obtained in Ref. [7]. Using the Selected- $G$ method with the slab potential, we have simulated the EEL spectra for slabs of few layers stacked graphene, according to Eq. (6). The parameters used in the calculations are given in table (II). We remind that the choice of the supercell is meaningless as our calculations do not depend on vacuum.

The results are presented in Fig. 5. They are in ex-

\begin{tabular}{lcccccc}
\hline \hline Parameters & 1L (R5) & 2L (R4) & 3L (R3) & 4L (R2) & 5L (R2) & 8L (R2) \\
\hline npwwfn & 2143 & 3451 & 3895 & 3451 & 4339 & 6931 \\
nbands $^{2}$ & 48 & 96 & 144 & 192 & 280 & 384 \\
npwmat $_{x y}$ & 19 & 19 & 19 & 19 & 19 & 19 \\
npwmat $_{z}$ & 7 & 13 & 19 & 25 & 31 & 49 \\
\hline
\end{tabular}

TABLE II. Parameters used to calculate the spectra of the slabs of different thicknesses.

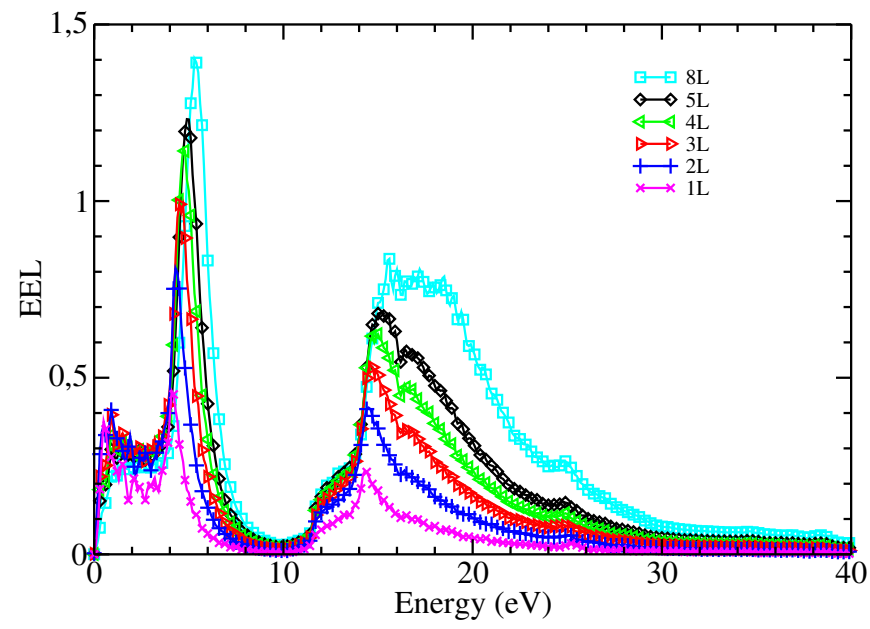

FIG. 5. (color online) Comparison of EELS spectra, including LF, for slabs of $N$ graphene layers $(N=1,2,3,4,5$ and 8$)$. Magenta with $\times: N=1$; blue with $+: N=2$; red with right triangle: $N=3$; green with left triangle: $N=4$; black with diamond: $N=5$; cyan with open square: $N=8$. In-plane component for $q_{\|}=10^{-2}$ a.u..

cellent agreement with the measured spectra of Ref. [7] unlike the spectra calculated with the untruncated external perturbation (see Appendix C 2). The energy position of each plasmon peak corresponds to the measured one. The progressive shift in energy when increasing the thickness and the spectral weight of the $\pi+\sigma$ plasmon are well reproduced. It shows a steep decrease above 14 $\mathrm{eV}$ for the thinner samples, which shifts towards higher energy with increasing thickness leading to a plateau, as observed in the experiment. Neverteless, the plateau is visible for the 8-layer slab, and not for the 5-layer one. To improve the agreement with the experimental results, we calculated EEL spectra for an in-plane momentum transfer of $q_{\|}=0.02$ a.u., for 1-layer, 2-layer and 5-layer slabs. Calculated spectra are shown in Fig. 6, where the measurements extracted from Ref. [7] are plotted in inset. The agreement is very good. The remaining difference appearing in the width of the spectra can be due either to the presence of the out-of-plane plasmon excitation, which is not included in our calculations, or, more probably, to the fact that, due to the collection angle, the experimental spectra come from the superposition of plasmons with different in-plane momentum transfers. Indeed, the plasmon dispersion leads to a blue shift of the spectra when the momentum transfer increases. 


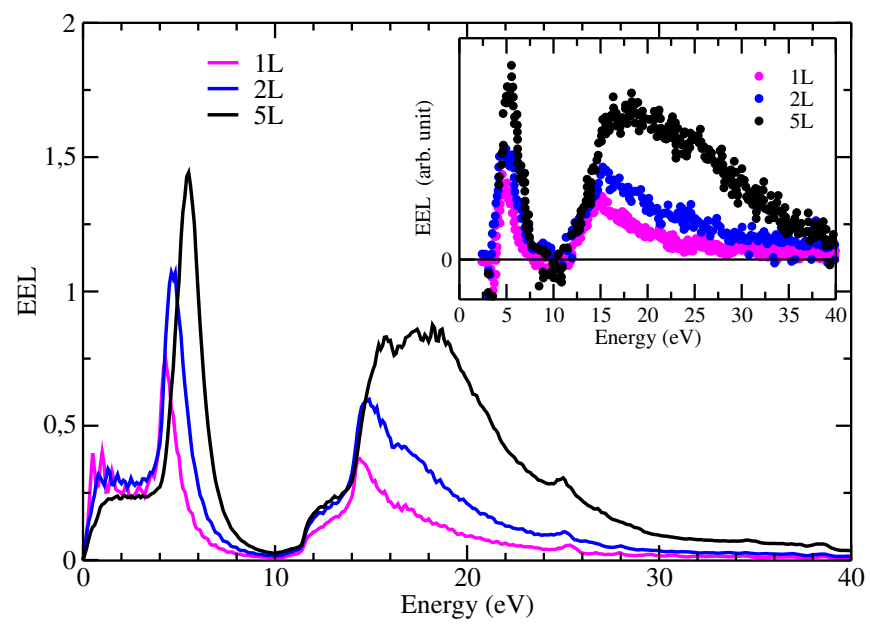

FIG. 6. (color online) Comparison of EELS spectra, for 1layer (magenta) 2-layer (blue) 5-layer (black) slabs. Calculations within Selected- $G$ and Slab potential framework for an in-plane momentum transfer $q_{\|}=2 \cdot 10^{-2}$ a.u..

\section{CONCLUSION}

In this paper, we calculated the EEL spectra for thin slabs of stacked graphene using TDDFT in reciprocal space in a supercell scheme. We evidenced that, unlike the absorption case, the influence of the vacuum, when using the standard supercell formalism, is dramatic for in-plane components. To solve this vacuum problem, we applied the Selected- $G$ approach, with a slab potential.

We obtained results independent of the vacuum intro- duced in the supercell, and in very good agreement with available measurements.

This independence of the spectra with respect to the vacuum can only be achieved thanks to the Selected- $G$ approach, where reciprocal lattice vectors are defined according to the thickness of the matter, and not the supercell, as it is usually done within the standard supercell formalism. This scheme is not limited to TDDFT, and could be applied to any other method involving the solution of a Dyson-type equation, such as the Bethe-Salpeter equation for instance. While the Selected- $G$ scheme is enough for the calculation of surface excitation spectra, as is was shown in Ref. 1, we emphasize here that the full expression of the slab potential must be taken into account to describe the EEL spectra of thin slabs.

We compared our formalism with other cutoff procedures developed earlier, showing that they can reproduce the shape of the spectra but with an amplitude depending on the choice of the supercell.

Our Selected- $G$ formalism, together with a slab potential, allows the accurate numerical simulations of EEL spectra using the efficient supercell-based formalism in reciprocal space. The question of the amplitude is a crucial issue, since the inverse microscopic dielectric matrix is also the key quantity for the calculation of the selfenergy in quasiparticles corrections.

\section{ACKNOWLEDGEMENT}

We would like to acknowledge GENCI (project 544) and CCRT-DRF for the computational support provided. We Thanks Lorenzo Sponza and Ralf Hambach for usefull discussions.

\section{Appendix A: Selected-G approach}

As the results presented in this paper are based on the Selected-G approach, we briefly sketch the main points of the derivation. For more details, one can refer to Ref. [1]

Assuming that the electronic density is localised in the $z$-direction between $-L_{z}^{\text {mat }}$ and 0 , we introduce two auxiliary periodic functions $\tilde{\chi}^{0}$ and $\tilde{\chi} \cdot \tilde{\chi}^{0}$ and $\tilde{\chi}$ are respectively identical to $\chi^{0}$ and $\chi$ for $\left(z, z^{\prime}\right)$ in $\left[-L_{z}^{m a t}, 0\right] \times\left[-L_{z}^{m a t}, 0\right]$, but the period is $L_{z}^{m a t}$, instead of $L_{z}$. Their Fourier transform is given by:

$$
\tilde{\chi}_{\tilde{\mathbf{G}}, \tilde{\mathbf{G}}^{\prime}}(\mathbf{q} ; \omega)=\frac{1}{L_{z}^{m a t}} \int_{-L_{z}^{m a t}}^{0} d z \int_{-L_{z}^{m a t}}^{0} d z^{\prime} e^{-i\left(q_{z}+\tilde{G}_{z}\right) z} \tilde{\chi}_{\mathbf{G}_{\| \mid}, \mathbf{G}^{\prime} \|}\left(\mathbf{q}_{\|}, z, z^{\prime} ; \omega\right) e^{i\left(q_{z}+\tilde{G}_{z}^{\prime}\right) z^{\prime}},
$$

where $\tilde{G}_{z}$ is defined according to the size of the material slab: $\tilde{G}_{z}=n \frac{2 \pi}{L_{z}^{m a t}}, n \in \mathbf{Z}$.

\section{Modified Dyson equation}

The Dyson equation linking the independent-particle response function and the microscopic susceptibility for an isolated slab is given by:

$$
\begin{aligned}
& \chi_{\mathbf{G}_{\|} \mathbf{G}_{\|}^{\prime}}\left(\mathbf{q}_{\|}, z, z^{\prime} ; \omega\right)=\chi_{\mathbf{G}_{\|} \mathbf{G}_{\|}^{\prime}}\left(\mathbf{q}_{\|}, z, z^{\prime} ; \omega\right) \\
& +\sum_{\mathbf{G}_{\|}^{\prime \prime}} \int_{-\infty}^{\infty} \int_{-\infty}^{\infty} d z_{1} d z_{2} \chi_{\mathbf{G}_{\|} \mathbf{G}_{\|}^{\prime \prime}}^{0}\left(\mathbf{q}_{\|}, z, z_{1} ; \omega\right) v_{\mathbf{G}_{\|}^{\prime \prime}}\left(\mathbf{q}_{\|}, z_{1}, z_{2}\right) \chi_{\mathbf{G}_{\|}^{\prime \prime} \mathbf{G}_{\|}^{\prime}}\left(\mathbf{q}_{\|}, z_{2}, z^{\prime} ; \omega\right)
\end{aligned}
$$


where the Fourier transforms for the periodic in-plane coordinates $(\|)$ has already been done. $v_{\mathbf{G}_{\| \mid}}\left(\mathbf{q}_{\|}, z, z^{\prime}\right)=$ $2 \pi e^{-\left|\mathbf{q}_{\|}+\mathbf{G}_{\|}\right|\left|z-z^{\prime}\right|} /\left|\mathbf{q}_{\|}+\mathbf{G}_{\|}\right|$is the 2 D Fourier transform of the 3D Coulomb potential.

Since i) $\chi_{\mathbf{G}_{\|} \mathbf{G}_{\|}^{\prime}}\left(\mathbf{q}_{\|}, z, z^{\prime} ; \omega\right)$ and $\chi_{\mathbf{G}_{\|} \mathbf{G}_{\|}^{\prime}}\left(\mathbf{q}_{\|}, z, z^{\prime} ; \omega\right)$ are equal to zero outside $\left[-L_{z}^{m a t}, 0\right] \times\left[-L_{z}^{m a t}, 0\right]$, and ii) equal respectively to $\tilde{\chi}^{0}$ and $\tilde{\chi}$ in this range, Eq. (A2) can be restricted to

$$
\begin{aligned}
& \tilde{\chi}_{\mathbf{G}_{\|} \mathbf{G}_{\|}^{\prime}}\left(\mathbf{q}_{\|}, z, z^{\prime} ; \omega\right)=\tilde{\chi}_{\mathbf{G}_{\|} \mathbf{G}_{\|}^{\prime}}\left(\mathbf{q}_{\|}, z, z^{\prime} ; \omega\right) \\
& +\sum_{\mathbf{G}_{\|}^{\prime \prime}} \int_{-L_{z}^{\text {mat }}}^{0} \int_{-L_{z}^{\text {mat }}}^{0} d z_{1} d z_{2} \tilde{\chi}_{\mathbf{G}_{\|}}^{0} \mathbf{G}_{\|}^{\prime \prime}\left(\mathbf{q}_{\|}, z, z_{1} ; \omega\right) v_{\mathbf{G}_{\|}^{\prime \prime}}\left(\mathbf{q}_{\|}, z_{1}, z_{2}\right) \tilde{\chi}_{\mathbf{G}_{\|} \mathbf{G}_{\|}^{\prime}}\left(\mathbf{q}_{\|}, z_{2}, z^{\prime} ; \omega\right)
\end{aligned}
$$

for $\left(z, z^{\prime}\right) \in\left[-L_{z}^{m a t}, 0\right] \times\left[-L_{z}^{m a t}, 0\right]$.

By Fourier-transforming equation (A3), one gets after some algebra:

$$
\tilde{\chi}_{\tilde{\mathbf{G}} \tilde{\mathbf{G}}^{\prime}}(\mathbf{q} ; \omega)=\tilde{\chi}_{\tilde{\mathbf{G}}^{\prime} \tilde{\mathbf{G}}^{\prime}}^{0}(\mathbf{q} ; \omega)+\sum_{\tilde{\mathbf{G}}_{1} \tilde{\mathbf{G}}_{2}} \tilde{\chi}_{\tilde{\mathbf{G}}^{\prime} \tilde{\mathbf{G}}_{1}}^{0}(\mathbf{q} ; \omega) \tilde{V}_{\tilde{\mathbf{G}}_{1} \tilde{\mathbf{G}}_{\mathbf{2}}}(\mathbf{q}) \tilde{\chi}_{\tilde{\mathbf{G}}_{2} \tilde{\mathbf{G}}^{\prime}}(\mathbf{q} ; \omega) .
$$

with $\tilde{\mathbf{G}}=\left(\mathbf{G}_{\|}, \tilde{G}_{z}\right), \mathbf{q}=\left(\mathbf{q}_{\|}, q_{z}\right)$ and where $q_{z}, \tilde{G}_{z}$ are vectors along the $z$ axis, defined according to $\tilde{G}_{z}=n_{z} \frac{2 \pi}{L_{z}^{\text {mat }}}$, with $n_{z} \in \mathbf{Z}$.

The slab potential $\tilde{V}$ is defined as

$$
\tilde{V}_{\tilde{G}_{1} \tilde{G}_{2}}\left(q_{z}\right)=\frac{1}{L_{z}^{\text {mat }}} \int_{-L_{z}^{\text {mat }}}^{0} \int_{-L_{z}^{\text {mat }}}^{0} d z_{1} d z_{2} e^{-i\left(\tilde{G}_{1}+q_{z}\right) z_{1}} v\left(z_{1}, z_{2}\right) e^{i\left(\tilde{G}_{2}+q_{z}\right) z_{2}}
$$

We finally get for the slab potential

$$
\begin{aligned}
& \tilde{V}_{\tilde{\mathbf{G}}_{1}, \tilde{\mathbf{G}}_{2}}(\mathbf{q})=\frac{4 \pi}{\left|\mathbf{q}+\tilde{\mathbf{G}}_{\mathbf{1}}\right|^{2}} \delta_{\tilde{\mathbf{G}}_{1}, \tilde{\mathbf{G}}_{2}} \\
& +\frac{4 \pi \xi \delta_{\mathbf{G}_{\mathbf{1}_{||}}, \mathbf{G}_{\mathbf{2}} \|}}{\left|\mathbf{q}+\tilde{\mathbf{G}}_{\mathbf{1}}\right|^{2}\left|\mathbf{q}+\tilde{\mathbf{G}}_{\mathbf{2}}\right|^{2}}\left[-\frac{e^{-\mid \mathbf{q}_{\|}+\mathbf{G}_{\mathbf{1}_{\|} \mid L_{z}^{\text {mat }}} \sin \left(q_{z} L_{z}^{m a t}\right)}}{L_{z}^{\text {mat }}}\left(2 q_{z}+\tilde{G}_{z 1}+\tilde{G}_{z 2}\right)\right) \\
& \left.+\frac{e^{-\left|\mathbf{q}_{||}+\mathbf{G}_{1 \| \mid}\right| L_{z}^{\text {mat }}} \cos \left(q_{z} L_{z}^{m a t}\right)-1}{L_{z}^{m a t}\left|\mathbf{q}_{\|}+\mathbf{G}_{\mathbf{1} \|}\right|}\left(\left|\mathbf{q}_{||}+\mathbf{G}_{\mathbf{1}||}\right|^{2}-\left(q_{z}+\tilde{G}_{z 1}\right)\left(q_{z}+\tilde{G}_{z 2}\right)\right)\right] .
\end{aligned}
$$

We assumed here that the matter is located between $-L_{z}^{m a t}$ and 0 . The same derivation can be conducted if the matter is located between $-L_{z}^{m a t} / 2$ and $L_{z}^{m a t} / 2$. In that particular case, a phase factor appears in the second term of the right-hand side of Eq. (A 6 ). This is accounted for by $\xi$, which can take the value 1 or $(-1)^{n_{1}+n_{2}}$ depending on the way the supercell is constructed. As before, $n_{i} \in \mathbf{Z}$ is defined according to $\tilde{G}_{z i}=n_{i} 2 \pi / L_{z}^{m a t}$.

This modified Dyson equation links the independent-particle response function and the susceptibility for the isolated slab, by means of the associated quantities of the periodic system. The modifications appear at two levels i) it requires a modified expression for the Coulomb potential (called Slab potential) and ii) the basis vector for the reciprocal space along the $z$ direction is $\tilde{G}_{z}=\frac{2 \pi}{L_{z}^{\text {mat }}}$ instead of $G_{z}=\frac{2 \pi}{L_{z}^{\text {supercell }}}$. For this reason we called it "Selected-G", where the selected $\mathbf{G}$ vectors are the ones of the matter and not the supercell.

\section{Inverse dielectric function}

Once we have defined the auxiliary functions, we can express the inverse dielectric function $\varepsilon^{-1}$ in terms of $\tilde{\chi}$.

By reducing the range of integration to $\left[-L_{z}^{m a t}, 0\right]$ in the following expression

$$
\varepsilon_{\mathbf{G}_{\| \mid}^{-1} \mathbf{G}_{\| \mid}^{\prime}}\left(\mathbf{q}_{\|}, z, z^{\prime} ; \omega\right)=\delta\left(z-z^{\prime}\right)+\int_{-\infty}^{\infty} d z_{1} v_{\mathbf{G}_{\| \mid}}\left(\mathbf{q}_{\|}, z, z_{1}\right) \chi_{\mathbf{G}_{\| \mid} \mathbf{G}_{||}^{\prime}}\left(\mathbf{q}_{\|}, z_{1}, z^{\prime} ; \omega\right)
$$

and using $\tilde{\chi}$ instead of $\chi$, we finally get

$$
\varepsilon_{\tilde{\mathbf{G}} \tilde{\mathbf{G}}^{\prime}}^{-1}(\mathbf{q} ; \omega)=\delta_{\tilde{\mathbf{G}} \tilde{\mathbf{G}}^{\prime}}+\sum_{\tilde{G}_{z 1}} \tilde{V}_{\tilde{\mathbf{G}} \tilde{\mathbf{G}}_{1}}(\mathbf{q}) \tilde{\chi}_{\tilde{\mathbf{G}}_{1} \tilde{\mathbf{G}}^{\prime}}(\mathbf{q} ; \omega)
$$

which corresponds to Eq. (6).

\section{Appendix B: Influence of the value of thickness of the matter}

The definition of the thickness of a $2 \mathrm{D}$ object is a very delicate point, which is far beyond the scope of this work.
Nevertheless, since it is a key quantity of our formalism, 
we have studied to which extend its value can influence the numerical results. We have chosen the case of the graphene sheet, which is one of the most critical case. Indeed, the incertainty regarding the extension of the electronic density will have a larger impact for graphene than for a thicker slab. Fig. 7 show the extension of the electronic density of the graphene sheet introduced in a cell of 31.47 Bohr. The red lines indicate the limits the thickness we have chosen (6.294 Bohr), resulting from the usual procedure, namely half of the $c$ parameter in the bulk graphite unit cell (after relaxation). This definition is closely related to the van der Waals atomic radius. ${ }^{41}$ According to Tables 1,7,8 and 9 of Ref. 39, the van der Waals radius of carbon atom spans in a range going from 1.5 to $1.96 \AA$. The blue lines in Fig. 7 show the thickness corresponding to the largest van der Waals radius $R_{v d W}$ $=1.96 \AA\left(L_{z}^{\text {graphene }}=7.408 \mathrm{Bohr}\right)$, which corresponds to an increase of $17 \%$ of the value used in our calculations.

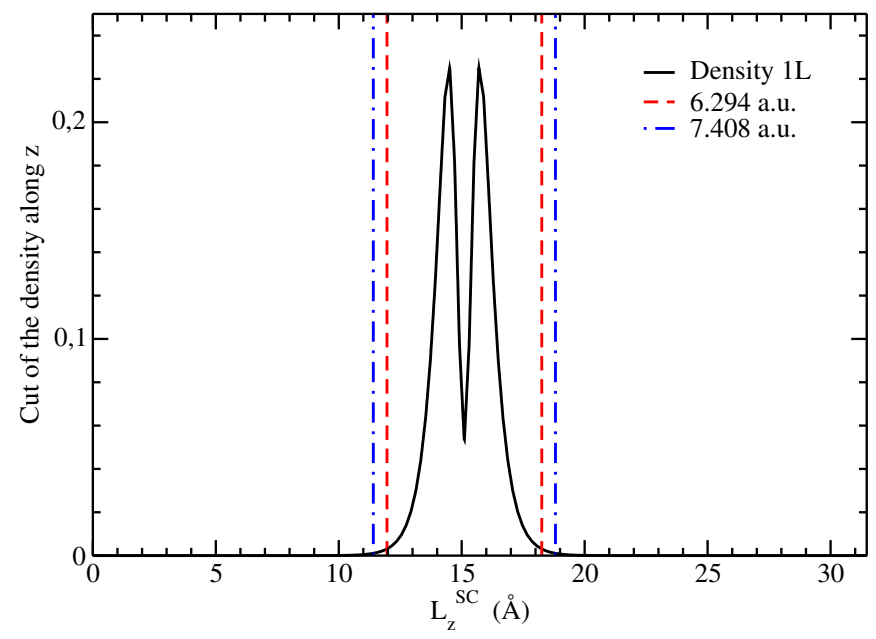

FIG. 7. (color online) (black line) Extension of the electronic density of one sheet of graphene, in a cell of 31.47 Bohr, (dashed red line) limits of $L_{z}^{\text {mat }}=6.294 \mathrm{Bohr}$, (dashed dotted blue line) limits of $L_{z}^{\text {mat }}=7.408$ Bohr.

To evaluate the influence of the thickness of the slab in our formalism, we have calculated the EELS spectra of the graphene layer using Selected- $G$ and the slab potential, considering three different values of $L_{z}^{m a t}$ : the two extreme ones (6.294 Bohr, 7.408 Bohr) and a intermediate value, $6.992 \mathrm{Bohr}$. It corresponds to $R_{v d W}=1.67$, 1.96 and $1.85 \AA$ respectively. The results are shown in Fig. 8 and Fig. 9 for $q_{\|}=10^{-2}$ a.u. and $q_{\|}=0.157$ a.u. respectively.

The spectra are superimposed, for each value of $q$. This shows that our results are not sensitive to the thickness used to define the matter in a range as large as $17 \%$ of $L_{z}^{m a t}$. This shows that, even though there is no perfectly unambiguous definition of the thickness of the layer, one can use safely standard definitions such as half of the $c$ parameter in the bulk unit cell or the van der Waals atomic radius.

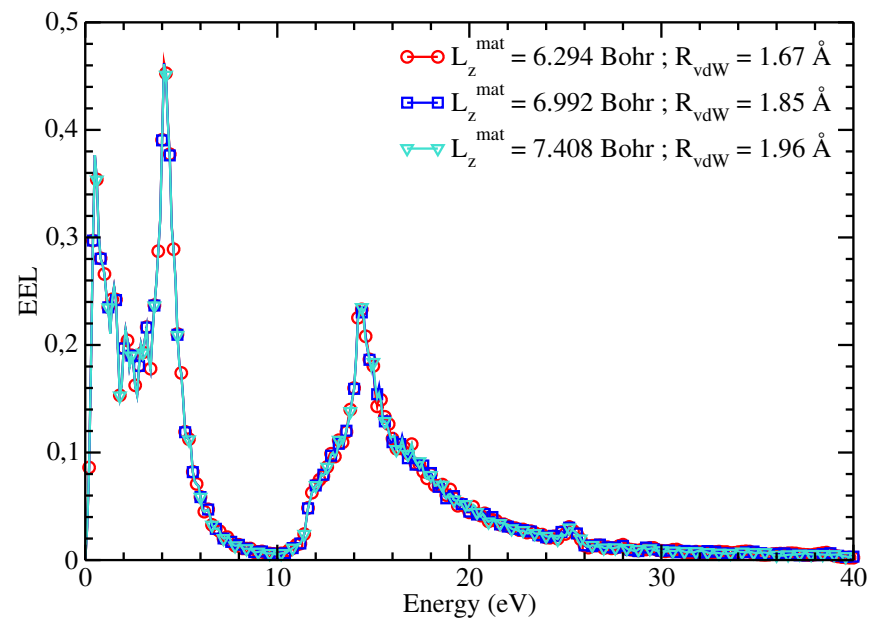

FIG. 8. (color online) Comparison of EELS spectra, including LF, within Selected- $G$ and slab potential framework, for one graphene layer depicted as a slab with different thicknesses. (Red with circle): $L_{z}^{\text {mat }}=6.294$ Bohr, (blue with square): $L_{z}^{\text {mat }}=6.992$ Bohr, (turquoise with down triangle): $L_{z}^{\text {mat }}=$ 7.408 Bohr. In-plane component for $q_{\|}=10^{-2}$ a.u..

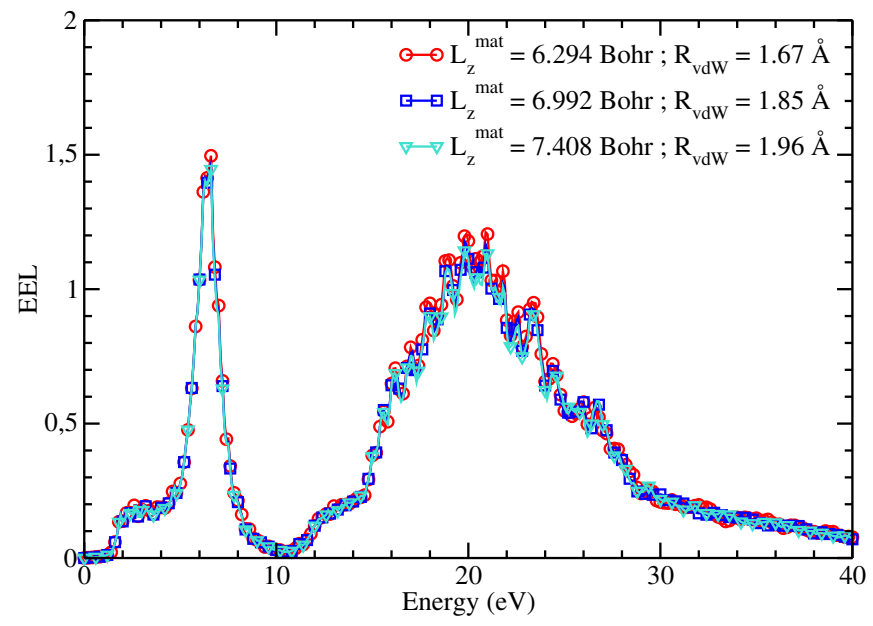

FIG. 9. (color online) Comparison of EELS spectra, including LF, within Selected- $G$ and slab potential framework, for one graphene layer depicted as a slab with different thicknesses. (Red with circle): $L_{z}^{\text {mat }}=6.294 \mathrm{Bohr,} \mathrm{(blue} \mathrm{with} \mathrm{square):}$ $L_{z}^{\text {mat }}=6.992$ Bohr, (turquoise with down triangle): $L_{z}^{\text {mat }}=$ 7.408 Bohr. In-plane component for $q_{\|}=0.157$ a.u.

\section{Appendix C: Truncated or untruncated external perturbation to calculate EELS ?}

\section{Influence of the vacuum for untruncated external perturbation}

For the case of the cutoff based on a diagonal expression of Fourier transform of the Coulomb potential $(\hat{v}),{ }^{26,27}$ it has been suggested ${ }^{24}$ that the EEL spectra should not be calculated by taking the imaginary part of $\varepsilon_{\mathbf{0 0}}^{-1}(\mathbf{q})=1+\hat{v}_{\mathbf{0}} \hat{\chi}_{\mathbf{0 0}}(\mathbf{q})$, as it results naturally from the evaluation of the screening, but by considering an expres- 
sion where the factor multiplying the response function is replaced by the untruncated external perturbation, leading to $\varepsilon_{\mathbf{0 0}}^{-1}(\mathbf{q})=1+v_{\mathbf{0}} \hat{\chi}_{\mathbf{0 o}}(\mathbf{q})$, where $v_{\mathbf{0}}=4 \pi / \mathbf{q}^{2}$. The Dyson equation giving $\hat{\chi}$ is solved with the cutoff potential $\hat{v}^{26,27}$

Since the purpose of part (IIID) was to compare spectra calculated with the cutoff potential $\hat{v},{ }^{26,27}$ with the ones calculated with Eq. (6) where the modified Coulomb potential is used, we plotted in part (IIID) spectra calculated according to $\varepsilon_{\mathbf{0 0}}^{-1}(\mathbf{q})=1+\hat{v}_{\mathbf{0}} \hat{\chi}_{\mathbf{0 0}}(\mathbf{q})$. In this appendix, we want to show that the spectra calculated with $\varepsilon_{\mathbf{0 0}}^{-1}(\mathbf{q})=1+v_{\mathbf{0}} \hat{\chi}_{\mathbf{0 0}}(\mathbf{q})$ also exhibit the vacuum problem.

The corresponding quantity for the non-diagonal form of the Fourier transform of the Coulomb potential would be:

$$
\varepsilon_{\mathbf{0 0}}^{-1}(\mathbf{q})=1+v_{\mathbf{0}} \tilde{\chi}_{\mathbf{0 0}}(\mathbf{q})
$$

where $\tilde{\chi}$ is the solution of Eq. (4).

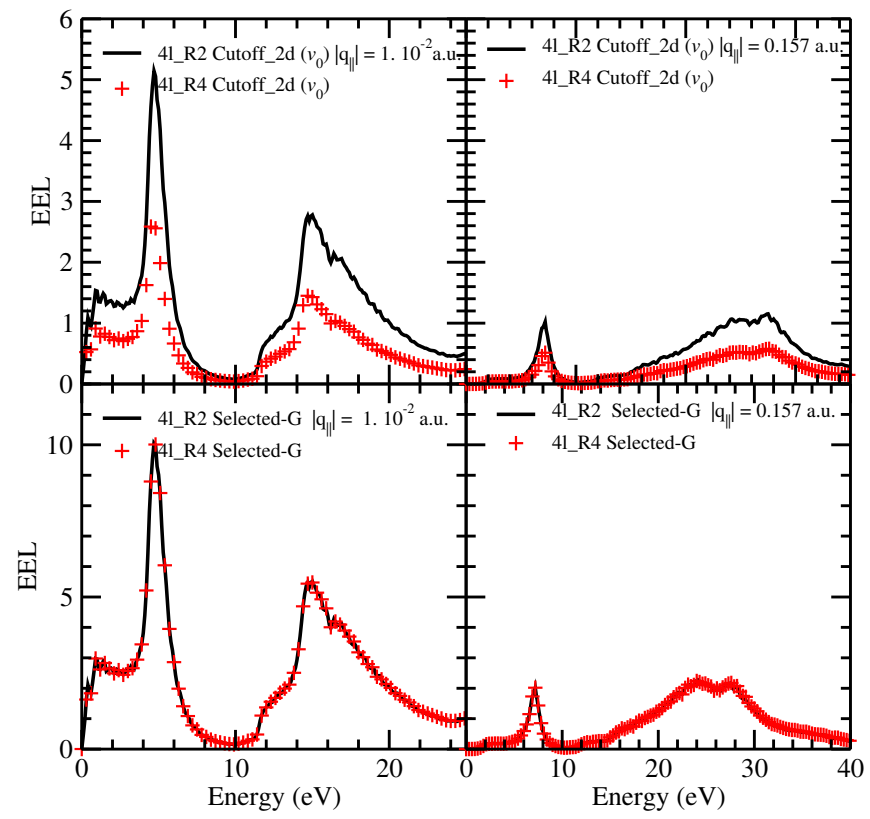

FIG. 10. (color online) Illustration of the vacuum effect for the different cutoff procedures on a 4 layer-graphene slab introduced into two supercells: ratio 2 (black continuous line) and ratio 4 (red crosses). (Top panel) cutoff proposed in Ref [26] and [27] with untruncated external potential in the factor of the response function; (Bottom panel) Selected-G + slab potential formalism. In-plane momentum transfer: (Left column) $q=1.10^{-2}$ a.u. and (right column) $q=0.157$ a.u..

The spectra are presented in Fig. (10). The top panel corresponds to the diagonal case, the bottom panel to the non-diagonal one. The spectra are larger than in Fig. (4). The spectra of the top panel are still dependent of the vacuum introduced in the supercell. The factor coming from the ratio of the heigth of the corresponding supercells occurs for the two range of $q$. It demonstrates that the effect is contained at the level of $\hat{\chi}$, and confirms the origin of the cancellation at vanishing $q$ shown in Fig. (4 - top panel). The spectra calculated using the Selected- $G$ procedure (Fig. 10 - bottom) to solve the Dyson equation are again independent of the vacuum introduced in the supercell.

Such results confirms that the Selected- $G$ procedure is the only method which provide spectra independent of vacuum. But they do not clarify if the external perturbation should be truncated or not, namely if one should calculate EELS according to Eq. (C1) or Eq. (6).

\section{Dependence with the thickness of the matter}

To remove the ambiguity concerning the use of the untruncated or truncated external perturbation, i.e. if one should use Eq. (C1) or Eq. (6) to calculate EELS, we will consider the EEL spectra calculated for slabs of different thicknesses, as in part (IIIE).

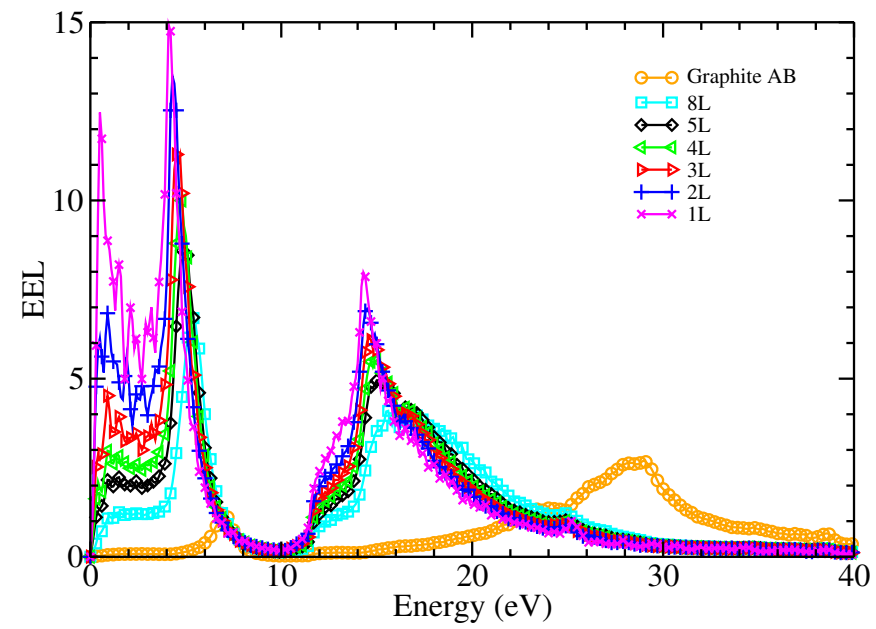

FIG. 11. (color online) Comparison of EELS spectra, including LF, for slabs of $N$ graphene layers ( $N=1,2,3,4,5$ and 8). Magenta with $\times: N=1$; blue with $+: N=2$; red with right triangle: $N=3$; green with left triangle: $N=4$; black with diamond: $N=5$; cyan with open square: $N=8$; orange with circle: graphite AB. In-plane component for $q_{\|}=10^{-2}$ a.u..

The spectra, calculated with Eq. (C1), are shown in Fig. (11). The evolution of the EEL spectra as the function of the thickness of the slab of matter is clearly nonphysical: the thinner the slab, the more intense the plasmon. This result allows us to confirm that the spectra must be calculated with Eq. (6), as shown in part (III E). 
Rev. B 92, 245308 (2015).

${ }^{2}$ R. F. Egerton, Reports on Progress in Physics 72, 016502 (2008).

3 M. Kociak, L. Henrard, O. Stéphan, K. Suenaga, and C. Colliex, Phys. Rev. B 61, 13936 (2000).

${ }^{4}$ C. Kramberger, R. Hambach, C. Giorgetti, M. H. Rümmeli, M. Knupfer, J. Fink, B. Büchner, L. Reining, E. Einarsson, S. Maruyama, F. Sottile, K. Hannewald, V. Olevano, A. G. Marinopoulos, and T. Pichler, Phys. Rev. Lett. 100, 196803 (2008).

${ }^{5}$ O. Stéphan, D. Taverna, M. Kociak, K. Suenaga, L. Henrard, and C. Colliex, Phys. Rev. B 66, 155422 (2002).

${ }^{6}$ G. G. Fuentes, E. Borowiak-Palen, T. Pichler, X. Liu, A. Graff, G. Behr, R. J. Kalenczuk, M. Knupfer, and J. Fink, Phys. Rev. B 67, 035429 (2003).

7 T. Eberlein, U. Bangert, R. R. Nair, R. Jones, M. Gass, A. L. Bleloch, K. S. Novoselov, A. Geim, and P. R. Briddon, Phys. Rev. B 77, 233406 (2008).

8 M. H. Gass, U. Bangert, A. L. Bleloch, P. Wang, R. R. Nair, and A. K. Geim, Nature Nanotechnology 3, 676 (2008).

${ }^{9}$ C. T. Pan, R. R. Nair, U. Bangert, Q. Ramasse, R. Jalil, R. Zan, C. R. Seabourne, and A. J. Scott, Phys. Rev. B 85, 045440 (2012).

10 P. Wachsmuth, R. Hambach, G. Benner, and U. Kaiser, Phys. Rev. B 90, 235434 (2014).

11 P. Wachsmuth, R. Hambach, M. K. Kinyanjui, M. Guzzo, G. Benner, and U. Kaiser, Phys. Rev. B 88, 075433 (2013).

12 S. C. Liou, C.-S. Shie, C. H. Chen, R. Breitwieser, W. W. Pai, G. Y. Guo, and M.-W. Chu, Phys. Rev. B 91, 045418 (2015).

13 M. J. Mohn, R. Hambach, P. Wachsmuth, C. Giorgetti, and U. Kaiser, Phys. Rev. B 97, 235410 (2018).

14 E. Runge and E. K. U. Gross, Phys. Rev. Lett. 52, 997 (1984).

15 S. Botti, A. Schindlmayr, R. D. Sole, and L. Reining, Rep. Prog. Phys. 70, 357 (2007).

16 Z. Yuan and S. Gao, Computer Physics Communications 180, 466 (2009).

17 M. Pisarra, A. Sindona, M. Gravina, V. M. Silkin, and J. M. Pitarke, Phys. Rev. B 93, 035440 (2016).

18 V. M. Silkin, E. V. Chulkov, and P. M. Echenique, Phys. Rev. Lett. 93, 176801 (2004).

19 G. P. Alldredge and L. Kleinman, Phys. Rev. Lett. 28, 1264 (1972).

20 M. Schlüter, J. R. Chelikowsky, S. G. Louie, and M. L. Cohen, Phys. Rev. Lett. 34, 1385 (1975).
21 O. Pulci, G. Onida, R. Del Sole, and A. J. Shkrebtii, Phys. Rev. B 58, 1922 (1998).

22 M. Palummo, G. Onida, R. Del Sole, and B. S. Mendoza, Phys. Rev. B 60, 2522 (1999).

23 J. R. Chelikowsky and M. L. Cohen, Phys. Rev. B 13, 826 (1976).

${ }^{24}$ R. Hambach, Theory and ab-initio calculations of collective excitations in nanostructures: towards spatially-resolved EELS, Ph.D. thesis (2010), Available at https://etsf.polytechnique.fr/system/files/ PhD_Hambach_2010.pdf.

25 I. Guilhon, M. Marques, L. K. Teles, M. Palummo, O. Pulci, S. Botti, and F. Bechstedt, Phys. Rev. B 99, 161201(R) (2019).

26 S. Ismail-Beigi, Phys. Rev. B 73, 233103 (2006).

27 C. A. Rozzi, D. Varsano, A. Marini, E. K. U. Gross, and A. Rubio, Phys. Rev. B 73, 205119 (2006).

${ }^{28}$ P. Cudazzo, I. V. Tokatly, and A. Rubio, Phys. Rev. B 84, 085406 (2011).

29 K. S. Thygesen, 2D Materials 4, 022004 (2017).

30 V. U. Nazarov, New Journal of Physics 17, 073018 (2015).

31 N. Tancogne-Dejean, C. Giorgetti, and V. Véniard, Phys. Rev. B 94, 125301 (2016).

${ }^{32}$ V. Despoja, D. Novko, K. Dekanić, M. Šunjić, and L. Marušić, Phys. Rev. B 87, 075447 (2013).

33 S. L. Adler, Phys. Rev. 126, 413 (1962).

34 N. Wiser, Phys. Rev. 129, 62 (1963).

35 G. Onida, L. Reining, and A. Rubio, Rev. Mod. Phys. 74, 601 (2002).

36 "The abinit code," The ABINIT code is a common project of the Université Catholique de Louvain, Corning Incorporated, and other contributors (URL http://www.abinit.org).

37 "The dp code," Theoretical Spectroscopy Group LSI - ETSF - Palaiseau; Available at https://etsf. polytechnique.fr/Software/Ab_Initio.

38 Z. H. Ni, H. M. Wang, J. Kasim, H. M. Fan, T. Yu, Y. H. Wu, Y. P. Feng, and Z. X. Shen, Nano Letters 7, 2758 (2007), pMID: 17655269, https://doi.org/10.1021/nl071254m.

39 S. S. Batsanov, Inorganic Materials 37, 871 (2001).

40 D. Novko, V. Despoja, and M. Šunjić, Phys. Rev. B 91, 195407 (2015).

41 L. Pauling, The Nature of the Chemical BondâAn Introduction to Modern Structural Chemistry. ((3rd edition) Ithaca, N.Y Cornell University Press, 1960). 